\title{
Hierarchical Segmentation of Thin Structures in Volumetric Medical Images
}

\author{
Michal Holtzman-Gazit ${ }^{1}$, Dorith Goldsher ${ }^{2}$, and Ron Kimmel ${ }^{3}$ \\ 1 Electrical Engineering Department \\ ${ }^{2}$ Faculty of Medicine - Rambam Medical Center \\ 3 Computer Science Department \\ Technion-I.I.T. Technion City, Haifa 32000, Israel
}

\begin{abstract}
We introduce a new method for segmentation of 3D medical data based on geometric variational principles. A minimal variance criterion is coupled with a geometric edge alignment measure and the geodesic active surface model. An efficient numerical scheme is proposed. In order to simultaneously detect a number of different objects in the image, a hierarchal method is presented. Finally, our method is compared with the multi-level set approach for segmentation of medical images.
\end{abstract}

Keywords: segmentation, active surfaces, energy minimization, level sets, variational methods, PDEs.

\section{Introduction and Previous Work}

Medical image analysis is the process through which trained personnel interpret visual medical information. In this paper we focus on imaging intracranial blood vessels as demonstrated on CT images. Our goal is to extract the blood vessels from these images and develop a tool that allows radiologists to rapidly view the vascular tree as a $3 \mathrm{D}$ object. Since demonstration of the bones surrounding the vessels enables radiologists to better localize the relevant vessels and better understand the anatomical relations between them, these bones are also included in our visualization tool.

The problem is that in CT Angiography images, the bones and the vessels usually have similar high density as compared to the brain parenchyma or, in other words, similar gray values. When thresholding an image that includes enhanced blood vessels as well as dense bones, they are usually extracted as the same object. Here, we present a hierarchical segmentation method using variational tools that allow us to extract bones and blood vessels as two separate $3 \mathrm{D}$ objects.

Codimension-two geodesic active contours were used in [20] for the segmentation of tubular structures. Region competition introduced in [31] were used in [11] for a similar purpose, while Fast Marching [27] and Level Sets methods were used in [10 12]. Edge alignment with the gradient field was used in [29] and [18]. While in [7/6], the segmentation methods are based on the piecewise constant Mumford-Shah model [25]. In [28] a hierarchical Mumford-Shah model is used to segment the image into more than two regions. 
Our model is based on geometric active contours [163,23,5,4,24,22] and level set methods [26]. It exploits efficient numerical schemes [2130] previously used for segmentation in [14 13]. Here we use the better qualities of previous methods in order to segment volumetric medical images. We combine the Chan-Vese's minimal variance method with a geometric edge alignment measure and the geodesic active surface model, and use an efficient numerical scheme for its implementation. Finally, we present a hierarchical method which allows us to simultaneously detect a number of objects in the image.

\section{Segmentation Methods - Variational Approach}

Minimal Variance and the Mumford-Shah Functional: In [7] the image is divided into two regions, interior and exterior. Their model minimizes the variance inside and outside the surface of the desired object. Given a 2D gray level image $I(x, y): \Omega \rightarrow \mathbb{R}^{+}$, Chan and Vese [7] proposed to use a minimal variance criterion given by the following functional,

$$
E_{M V}\left(C, c_{1}, c_{2}\right)=\iint_{\Omega_{C}}\left(I-c_{1}\right)^{2} d x d y+\iint_{\Omega \backslash \Omega_{C}}\left(I-c_{2}\right)^{2} d x d y,
$$

where $C$ is the contour separating the two regions and $\Omega_{C}$ is the area inside the contour. When minimizing this functional $c_{1}$ and $c_{2}$ obtain the mean intensity values of the image in the interior and the exterior parts of the curve $C$, respectively. The optimal curve would best separate the interior and the exterior with respect to their relative expected values.

In [6] this model was extended to the general piecewise constant Mumford-Shah model [25]. It minimizes the following functional,

$$
E_{M S}=\sum_{i} \iint_{R_{i}}\left(I-\text { mean }_{R_{i}} I\right)^{2} d x d y+\nu_{0}|\Gamma|,
$$

where $R_{i}$ are the non-overlapping regions, and $|\Gamma|$ measures the arc length of the separating contour and thus controls its smoothness.

A straightforward level set representation associates one function to each region. Thus, Chan and Vese proposed to use $n$ functions in order to define up to $2^{n}$ regions. Each function defines two non-overlapping regions, $\left\{\Phi_{n}>0\right\}$ and $\left\{\Phi_{n}<0\right\}$. All the binary combinations between the positive and negative regions of each of the $n$ functions, define $2^{n}$ non-overlapping regions. This method was extended and implemented for 3D images in [6].

Edge based techniques: Given a gray level image $I(x, y): \mathbb{R}^{2} \rightarrow[0,1]$, we define the gradient direction vector field $\boldsymbol{\xi}(x, y)=\frac{\nabla I}{|\nabla I|}$, and the orthogonal vector field $\boldsymbol{\eta}(x, y)=$ $\frac{\bar{\nabla} I}{|\nabla I|}$, so that $\langle\boldsymbol{\xi}, \boldsymbol{\eta}\rangle=0$. The Haralik edge detector [152] finds the image locations where both $|\nabla I|$ is greater than some threshold and $I_{\xi \xi}=0$.

We would like to propagate an initial contour that stops along the desired object's boundaries. For that, we need a geometric functional that yields $I_{\xi \xi} \boldsymbol{n}=0$ as an EulerLagrange (EL) equation, where $\boldsymbol{n}$ is the unit normal to the curve. In [19] the authors used the fact that $I_{\xi \xi}=\Delta I-I_{\eta \eta}$ to show that the maximization of the functional

$$
\int_{\partial \Omega_{C}}\langle\nabla I, \boldsymbol{n}\rangle d s-\iint_{\Omega_{C}} \kappa_{I}|\nabla I| d x d y
$$


yields $I_{\xi \xi} \boldsymbol{n}=0$. Here, $\kappa_{I}$ is the curvature of the level sets of the image. Thereby, the above functional maximizes the alignment between the image gradient and the edge normals while minimizing topological complexity of the image inside the curve.

In order to extend this idea to three dimensions it can be shown that $I_{\xi \xi}=\Delta I-$ $H_{I}|\nabla I|$, where $H_{I}$ is the mean curvature of the level sets of the image. In this case, the level sets are surfaces in the volumetric image data. Therefore, the functional that generates a similar flow in 3D has two parts:

1. Maximizing the geometric integral measure $\iint_{S}\langle\nabla I, \boldsymbol{n}\rangle d a$, where $S(u, v)$ is the evolving surface, $d a$ is the surface area element, and $\boldsymbol{n}$ is the unit normal to the surface. The maximization of this functional yields $\Delta I \boldsymbol{n}=0$.

2. Minimizing the functional $\iiint_{\Omega_{S}} H_{I}|\nabla I| d x d y d z$. Where $\Omega_{S}$ is the volume inside the surface $S(u, v)$. By Green's theorem, the EL equations are $H_{I}|\nabla I| \boldsymbol{n}=0$.

Therefore, the energy functional that yields $I_{\xi \xi} \boldsymbol{n}=0$, is given by

$$
E_{C}(S)=\iint_{S}\langle\nabla I, \boldsymbol{n}\rangle d a-\iiint_{\Omega_{s}} H_{I}|\nabla I| d x d y d z .
$$

This term is very important in finding the edges of the thin structures in volumetric medical images. It has the ability to track edges of a thin low contrast object.

Geodesic Active Surface: The geodesic active surfaces model [54] is defined by the functional

$$
E_{G A C}(S)=\iint g(S) d a,
$$

where $d a$ is the surface area element and $g(x, y, z)$ is an inverse edge indicator function given, for example, by $g(x, y, z)=\frac{1}{1+\left|\frac{\nabla I}{\alpha}\right|^{2}}$.

Minimizing this functional is a surface along which $g$ obtains the smallest possible values. The EL equation of this functional is $(g H-\langle\nabla g, \boldsymbol{n}\rangle) \boldsymbol{n}=0$, where $H$ is the mean curvature of the surface $S$ and $\boldsymbol{n}$ is the normal to the surface. By setting $g$ to a constant, the functional measures the surface area. The EL of such a functional defines a minimal surface for which the mean curvature is equal to zero. This type of functional was used by Chan and Vese as a regularization, in order to control the smoothness of the evolving surface.

In our scheme, the geodesic active surface is used for regularization. Its added value over the mean curvature flow is its sensitivity to the actual edge via the function $g$, which guides the boundary surface towards the desired object's boundaries.

\section{Proposed Functional and Level Set Formulation}

We propose a functional which is a weighted sum of the terms discussed in the previous sections.

$$
E_{\text {Total }}=-E_{C}+\beta E_{M V}+\gamma E_{G A C},
$$

where $\beta, \gamma$ are positive constants. The surface evolution towards an extremum derived from this functional is given by

$$
S_{t}=\left(-I_{\xi \xi}-\beta\left(\left(I-c_{1}\right)^{2}-\left(I-c_{2}\right)^{2}\right)+\gamma(g H-\langle\nabla g, \boldsymbol{n}\rangle)\right) \boldsymbol{n} .
$$


Next, we embed a closed surface in a higher dimensional $\Phi(x, y, z)$ function, which implicitly represents the surface $S$ as a zero level set, i.e. $S=\{\{x, y, z\} \mid \Phi(x, y, z)=0\}$. This is the Osher Sethian level set formulation [26] for the evolution of surfaces. Given a surface evolution $S_{t}=\gamma \boldsymbol{n}$, the implicit level set evolution reads $\Phi_{t}=\gamma|\nabla \Phi|$.

The level set formulation of our proposed surface evolution equation is thereby

$$
\Phi_{t}=\left(-I_{\xi \xi}-\beta\left(\left(I-c_{1}\right)^{2}-\left(I-c_{2}\right)^{2}\right)+\gamma\left(\operatorname{div}\left(g \frac{\nabla \Phi}{|\nabla \Phi|}\right)\right)\right)|\nabla \Phi|
$$

Hierarchical Method: In order to extract more than one object from the image, we use a hierarchical method. In each stage we choose one subregion that includes more than one object, and divide it into two subregions. For a given image, we first apply the segmentation algorithm described in the previous sections. At the end of this stage we have a surface that describes the edges of the segmented object. If there is a need for further segmentation, we manually choose one of the regions generated from the previous step and apply the segmentation algorithm again only to this region. This way, we segment only the interesting parts of the image.

\section{Numerical Scheme}

If $\Phi(x, y, z ; t)$ is a distance function of the surface $S(t)$, we can simplify the short time evolution equation by setting $|\nabla \Phi|=1$. Distance computation can be done by using the fast marching algorithm [27]. We also use a narrow band approach [1,8], where in every iteration we compute the distance only at voxels close to the zero set. By this approach we have a computationally efficient explicit scheme. However, an explicit scheme works with a small limited time step due to stability issues.

In order to construct an unconditionally stable scheme we use a locally one dimensional (LOD) scheme suggested in [1730]

$$
\begin{aligned}
\Phi^{n+1} & =\prod_{i=1}^{3}\left(\mathcal{I}-\tau \gamma A_{i}\right)^{-1}\left(\Phi^{n}+\tau f\right) \\
f & =-\left(\beta\left(\left(I-c_{1}\right)^{2}-\left(I-c_{2}\right)^{2}\right)+I_{\xi \xi}\right),
\end{aligned}
$$

where $\mathcal{I}$ is the identity matrix, $\tau$ is the time step, and $\beta$ and $\gamma$ are constants. $A_{i}$ are tridiagonal matrix operators given by $A_{i}=\frac{\partial}{\partial i} g \frac{\partial}{\partial i}$, where $i=x, y, z$. The LOD scheme is used in order to accelerate the propagation of the surface in a stable way. This is due to the fact that we can use a time step of any size, and therefore the scheme converges efficiently. For an image of size $100^{3}$ voxels the program runs a few minutes on a Pentium III PC using double precision.

\section{Experimental Results}

Let us present the segmentation results of our algorithm using the hierarchical approach and compare it to the Chan-Vese multi-level set algorithm. Figures 10 - 2 show 3D hierarchical segmentation of CT angiography (CTA) images of the brain. We applied the 
first step of our algorithm to a 3D image which is a part of the full CT image. The MIP (maximal intensity projection) of this image is shown in Figure 17a) and a 2D slice of this image is shown in Figure 1 (b). In this image the vessels appear as light gray while the bones appear as white. We initialized the surface as a small balloon inside one of the blood vessels and allowed it to grow towards the boundaries. The result of the first segmentation phase is shown in Figure 2 (a). The algorithm captured the bright parts of the image which include the bones and the blood vessels. In order to split this object into two separate objects we applied the second step of our algorithm only to the region generated by the first step. The results are shown in Figure 2(b).

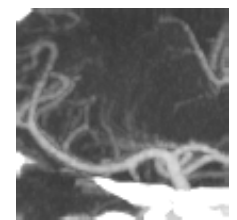

(a)

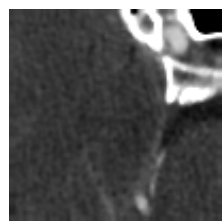

(b)

Fig. 1. (a) MIP of a $100^{3}$ volume of a CTA image of the brain. (b) A $100^{2}$ part of a $2 \mathrm{D}$ CTA image of the brain. The bones adjacent to the brain appear as white, and the blood vessels appear as light gray.

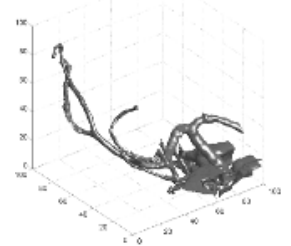

(a)

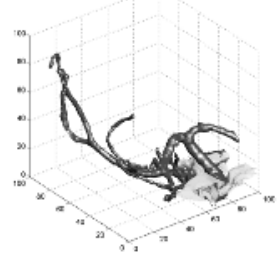

(b)

Fig. 2. (a) First phase segmentation result on the CTA image. (b) Hierarchical segmentation results on the CTA image. The yellow surface shows the bone and the red surface shows the blood vessels.

There are some cases where the multi-level set algorithm does not identify the regions correctly. The problem occurs when the image consists of adjacent objects which have close gray values and a certain amount of noise. Since the adjacent objects have similar gray values, the multi-level set algorithm identifies three regions instead of two. Our hierarchical algorithm, on the other hand, works in two steps. It first isolates the two objects from the background, and then differentiates between them. Therefore, in the second stage of the algorithm we are left with only two regions to segment. A synthetic example is shown in Figure 3.

We applied Chan-Vese's multi-level-set algorithm (using two level sets) to a part of a 3D CT angiography image of the brain. The algorithm generated three regions and background (i.e. four regions). The three foreground regions are shown in Figure 5 and a contour of each region on a 2D slice of the image is shown in Figure 6 In this case we do not have a single object that depicts the blood vessels. Part of the blood vessels were classified as one object Figure 5 a), while other parts of the blood vessels were classified as a different object that includes parts of the bone, Figure 5(c). A third object included most of the bone data Figure 5b). This result is due to the fact that the blood vessel data appears in varying levels of light gray. Therefore, the algorithm generates four different regions instead of three.

Next, we compared these results to our hierarchical algorithm. We applied the first step of the algorithm to the whole image. It generates an object consisting of the blood vessels and the bones. The second step generated two objects as shown in Figure 4 In 


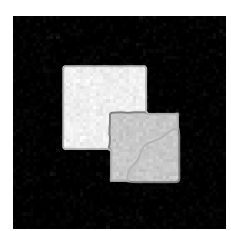

(a)

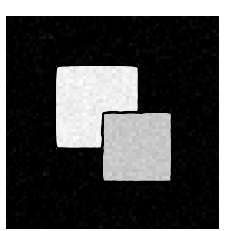

(b)

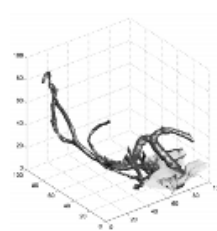

(a)

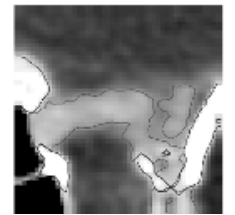

(b)
Fig. 3. (a) Shows the results of Chan-Vese's multi-level-set segmentation algorithm. The two zero sets are painted in red and green. (b) Shows the result of our hierarchical algorithm. The final objects are bounded by red and blue contours
Fig. 4. (a) The results of our hierarchical algorithm on the 3D CT image of the brain. The yellow surface depicts the bone data while the red surface depicts the vessels. (b) A 2D slice of the CT data of the brain showing the contours of the two objects generated by our segmentation algorithm.

this case we generated only two objects, because we first segment only the bright pixels from the whole data, and afterwards divide this data to darker (vessels) and brighter parts (bones). We control the level of detail we want to give each of the regions in the first step. This also allows us to change the parameters of the algorithm in each step in order to capture the different parts of the image.

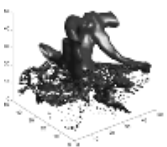

(a)

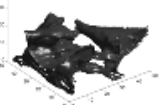

(b)

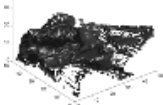

(c)

Fig. 5. The three foreground regions generated by Chan-Vese's algorithm using two level set function
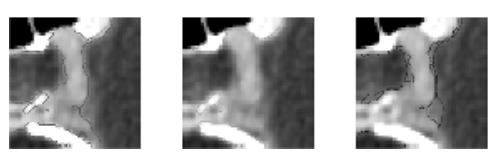

Fig. 6. A 2D slice of the CT image showing the three foreground regions generated by Chan-Vese's algorithm using two level set functions. The contours in each frame represent the different regions

When dealing with MRI images of the brain, we have a similar problem of segmenting the gray matter and the white matter as two different objects. Figure 7 shows the segmentation result of our hierarchical segmentation versus the Chan-Vese two level set approach of a synthetic 3D MRI image generated by the BrainWeb [9]. The results are similar, however there are some locations where our hierarchical approach better segments between the white matter and the gray matter. The two level set approach includes regions classified as a third object which is neither white nor gray. Figure 8 shows a 3D object which is the gray matter generated by our segmentation algorithm. 


\section{Conclusions}

We introduced a new segmentation method of 3D medical images. We proposed an efficient numerical scheme for this method. Next, we used it hierarchically in order to segment few objects from the image. Finally, we compared our results with the ChanVese multi-level-set method. In cases where the intensity levels of the different objects were close, we found that our hierarchical approach better segments the desired objects than the Chan-Vese multi-level-set approach.

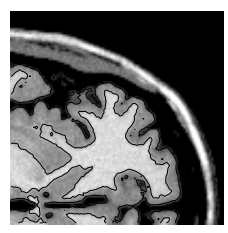

(a)

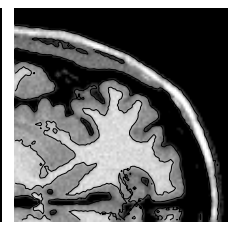

(b)

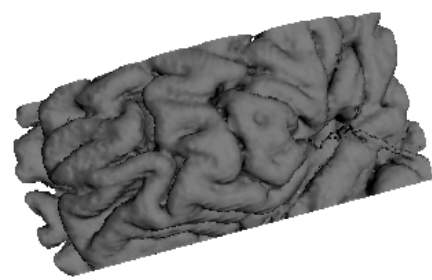

Fig. 7. (a) Hierarchical segmentation, first phase - red contour, second phase - blue contour. (b) Two level set approach of ChanVese. The blue and red contours are the zero Fig. 8. The result of our segmentation algorithm on a $3 \mathrm{D}$ MRI image. level of each level set.

\section{References}

1. D. Adalsteinsson and J.A. Sethian. A fast level set method for propagating interfaces. J. of Comp. Phys., 118:269-277, 1995.

2. J. Canny. A computational approach to edge detection. IEEE Trans. on PAMI, 8(6):679-698, 1986.

3. V. Caselles, F. Catte, T. Coll, and F. Dibos. A geometric model for active contours. Numerische Mathematic, 66:1-31, 1993.

4. V. Caselles, R. Kimmel, G. Sapiro, and C. Sbert. Minimal surfaces based object segmentation. IEEE Trans. on PAMI, 19(4):394-398, 1997.

5. V. Casseles, R. Kimmel, and G. Sapiro. Geodesic active contours. IJCV, 22(1):61-79, 1997.

6. T. Chan and L. Vese. Active contour and segmentation models using geometric PDE's for medical imaging. UCLA Computational and applied mathematics reports, December 2000.

7. T. Chan and L. Vese. Active contours without edges. IEEE Trans. on IP, 10(2):266 -277, 2001.

8. D. L. Chopp. Computing minimal surfaces via level set curvature flow. J. of Computational Physics, 106(1):7791, May 1993.

9. C.A. Cocosco, V. Kollokian, R.K.S. Kwan, and A.C. Evans. Brainweb: Online interface to a 3D MRI simulated brain database. NeuroImage, vol.5, no.4, part 2/4, S425 in Proceedings of 3rd International Conference on Functional Mapping of the Human Brain, May 1997. Copenhagen, http://www.bic.mni.mcgill.ca/brainweb/. 
10. L. D. Cohen and R. Kimmel. Global minimum for active contours models: A minimal path approach. IJCV, 24(1):57-78, 1997.

11. T. Deschamps. Curve and shape extraction with minimal path and level sets techniques. Applications to 3D medical imaging. PhD thesis, Univesity of Paris Daphine, 2001.

12. T. Deschamps and L.D. Cohen. Fast extraction of tubular and tree $3 \mathrm{~d}$ surfaces with front propagation methods. In Proc. of 16th ICPR, 1:731 -734, August 2002.

13. R. Goldenberg, R. Kimmel, E. Rivlin, and M. Rudsky. Cortex segmentation - a fast variational geometric approach. IEEE Trans. on Medical Imaging, 21(2):1544-1551, 2002.

14. R. Goldenberg, R.Kimmel, E. Rivlin, and M.Rudsky. Fast geodesic active contours. IEEE Trans. on IP, 10(10):1467-1475, 2001.

15. R. Haralik. Digital step edges from zero crossing of second directional derivatives. IEEE Trans. on PAMI, 6(1):58-68, January 1984.

16. M. Kass, A. Witkin, and D. Terozopolous. Snakes: Active contour models. IJCV, 1(4):321331, 1988.

17. R. Kimmel. Fast edge integration. In S. Osher and N. Paragios, editors, Geometric Level Set Methods in Imaging, Vision and Graphics. Springler Verlag, 2002.

18. R. Kimmel and A.M. Bruckstein. Regularized laplacian zero crossings as optimal edge integrators. Proc. of Image and Vision computing, IVCNZ01, November 2001.

19. R. Kimmel and A.M. Bruckstein. On edge detection edge integration and geometric active contours. IEEE Trans. on PAMI, submitted.

20. L.M. Lorigo, O. Faugeras, W. Grimson, R. Keriven, R. Kikinis, C.F. Westin, and Ayra Nabavi. Codimention-two geodesic active contours for the segmentation of tubular structures. Proc. of CVPR, 2000.

21. T. Lu, P. Neittaanmaki, and X-C. Tai. A parallel splitting up method and its application for navier-stokes equations. Applied Mathematics Letters, 4(2):25-29, 1991.

22. R. Malladi and J.A. Sethian. An o(n $\log (\mathrm{n}))$ algorithm for shape modeling. in Proceedings of National Academy of Sciences, USA, 93:9389-9392, Sept 1996.

23. R. Malladi, J.A. Sethian, and B.C. Vermuri. Shape modeling with front propagation: A level set approach. IEEE Trans. on PAMI, 17(2):158-175, Feb. 1995.

24. T. McInerney and D. Terzopoulos. Topology adaptive deformable surfaces for medical image volume segmentation. IEEE Trans. on Medical Imaging, 18(10):840 -850, October 1999.

25. D. Mumford and J. Shah. Optimal approximation by piecewise smooth functions and associated variational problems. Comm. Pure Applied Math, 42:577-685, 1989.

26. S.J. Osher and J. A. Sethian. Fronts propagating with curvature dependent speed: Algorithms based on hamilton jacobi formulations. Journal of Com. Phys., 79:12-49, 1988.

27. J. A. Sethian. Level Set Methods: evolving interfaces in geometry, fluid mechanics, computer vision, and materials science. Cambridge University Press, Cambridge, 1996.

28. A. Tsai, A. Yezzi, and A.S. Willsky. Curve evolution implementation of the mumford shah functional for image segmentation, denoising, interpolation and magnification. IEEE Trans. on IP, 10(8):1169- 1186, 2001.

29. A. Vasilevskiy and K. Siddiqi. Flux maximizing geometric flows. IEEE Trans. on PAMI, 24(12):1565-1578, December 2002.

30. J. Weickert, B.M. ter Haar Romeny, and M.A. Viergever. Effiecient and reliable scheme for nonlinear diffusion filtering. IEEE Trans. on IP, 7(3):398-410, 1998.

31. S. Zhu and A.Yuille. Region competition: Unifying snakes, region growing, energy/Bayes/MDL for multi-band image segmentation. IEEE Trans on PAMI, 19(9), 1996. 